\title{
Insulin Resistance and Its Associated Risk Factors in Nigerian Women with Polycystic Ovary Syndrome
}

\author{
Chinyere B. N. Akpata1 ${ }^{*}$, Patrick O. Uadia ${ }^{1}$, Friday E. Okonofua ${ }^{2}$ \\ ${ }^{1}$ Department of Biochemistry, University of Benin, Benin City, Nigeria \\ ${ }^{2}$ Department of Obstetrics and Gynecology, University of Medical Sciences, Ondo, Ondo State, Nigeria \\ Email: *cbnakpata@gmail.com
}

How to cite this paper: Akpata, C.B.N., Uadia, P.O. and Okonofua, F.E. (2019) Insulin Resistance and Its Associated Risk Factors in Nigerian Women with Polycystic Ovary Syndrome. Open Journal of Obstetrics and Gynecology, 9, 382-394. https://doi.org/10.4236/ojog.2019.93039

Received: February 26, 2019

Accepted: March 15, 2019

Published: March 18, 2019

Copyright $\odot 2019$ by author(s) and Scientific Research Publishing Inc. This work is licensed under the Creative Commons Attribution International License (CC BY 4.0).

http://creativecommons.org/licenses/by/4.0/

\begin{abstract}
Background: The etiology of polycystic ovary syndrome (PCOS) is not completely understood; however one condition that correlates closely with the pathogenesis of PCOS is insulin resistance (IR). The objective of this study was to determine the prevalence of insulin resistance (IR) and the association of such abnormality with potential risk factors in women with PCOS. Method: 116 women with confirmed PCOS attending a reproductive clinic at the University of Benin Teaching Hospital in Benin City were studied. IR was determined by homeostatic model assessment (HOMA) $\geq 2$ and pre-diabetes by fasting plasma glucose between 110 and $125 \mathrm{mg} / \mathrm{dl}$ and/or plasma glucose value between 140 and $200 \mathrm{mg} / \mathrm{dl}$ at 2 hours during an oral glucose tolerance test (OGTT) after ingestion of $75 \mathrm{~g}$ oral glucose load. Results: Forty-two women were insulin resistant among the 116 women with PCOS. The prevalence of IR was $36.2 \%$ ( $95 \%$ CI 26.6 - 46.2). The prevalence of impaired fasting glucose (IFG) showed 1.7\% (95\% CI 0.97 - 2.03), impaired glucose tolerance (IGT) was $2.6 \%$ (95\% CI 1.97 - 3.03) and diabetes mellitus (DM) was $1.7 \%$ (95\% CI $0.97-2.03$ ) in the 116 PCOS women. Of these 42 insulin resistant PCOS women, 23.8\% $(n=10)$ were obese and $40.5 \%(n=17)$ were overweight. Multivariate analysis revealed that total cholesterol (OR, 1.07; 95\% CI, $1.04-1.10$ ), triglycerides (OR, 1.08; 95\% CI, $1.04-1.13$ ) and LDL-cholesterol (OR, 1.08; 95\% CI, 1.04 - 1.12) were statistically significant independent risk factors for IR. Conclusion: The prevalence of IR was high in women with PCOS, and there was a significant association between IR, obesity, and dyslipidemia. However, the prevalence rates of impaired glucose tolerance and DM were low in women with PCOS compared to other studies. Since women with PCOS are at risk of IR and dyslipidemia, early screening, detection, intervention, and lifestyle modification would ameliorate the financial burden of DM and cardiovascular disease (CVD).
\end{abstract}




\section{Keywords}

Impaired Glucose Tolerance, Insulin Resistance, Nigerian Women, Polycystic Ovary Syndrome, Risk Factors

\section{Introduction}

Polycystic ovary syndrome (PCOS) is the most common endocrine disorder affecting women in childbearing age. It is a heterogeneous disorder whose pathogenesis is not well understood despite evidence of complex interaction with genetic, behavioral and environmental factors that contribute to its occurrence [1]. The manifestations of PCOS are not confined to the gynecological sphere. Women afflicted by this disorder show an increased prevalence of several co-morbidities, including obesity, dyslipidemia, hypertension, metabolic syndrome (MS), and type 2 diabetes mellitus (T2DM) in comparison with women without PCOS, and these features underlie the greater risk of developing cardiovascular disease (CVD) [2]. However, one condition that correlates closely with the pathogenesis of PCOS is insulin resistance, which is present in $50 \%-75 \%$ of women with PCOS [3]. Insulin resistance and subsequent compensatory hyperinsulinemia which are closely related to the pathogenicity and co-morbidities of PCOS can be exacerbated by the coexistence of obesity which affects approximately $50 \%$ of PCOS women [4]. The prevalence rates of IR reported among US women with PCOS range from $44 \%$ to $70 \%$ [3] which are significantly higher than the rates reported among Iranian, Thai, and Vietnamese PCOS women [5] [6] [7]. Reyes-Munoz et al. (2016) [8], reported IR prevalence of $60.2 \%$ among infertile Mexican women with PCOS, a rate that was higher in overweight and obese women than in normal-weight women. Some studies have reported independent risk factors associated with IR in PCOS women to include age, BMI, waist circumference (WC), waist-to-hip ratio (WHR), the presence of acanthosis nigricans, dyslipidemia, and obesity [6] [7] [8]. Wanderley et al. (2018) [9] however, reported lipid accumulation product (LAP) and arterial hypertension (AH) as additional risk factors besides WC, BMI, and dyslipidemia in a high proportion of patients in their study. Considering that the prevalence rate of PCOS among Nigerian women is as high as $27.6 \%$ [10] and that no such study has been conducted to the best of our knowledge in this region, there is the need to provide data on the prevalence of IR in our population for improved clinical decision making. The present study aims at determining the prevalence of IR and the association of such abnormalities with potential risk factors in women with PCOS.

\section{Methods}

A sample of women attending the infertility clinics at the University of Benin Teaching Hospital (UBTH) and the Women's Health and Action Research Cen- 
tre (WHARC) in Benin City, Nigeria between April 1, 2009, and November 30, 2010, formed the subject of the study. Among the women, 116 (aged $18-45$ years) were diagnosed with PCOS according to the 2003 Rotterdam criteria [10]. After fully informed consent was obtained from the women, a pre-prepared standard proforma was used to obtain data on their age, clinical and medical history, anthropometric and blood pressure measurements, and hirsutism/skin manifestations. The detailed method of clinical examination has been published elsewhere [10].

Glucose tolerance was evaluated using a $75 \mathrm{~g}$ oral glucose tolerance test (OGTT). After an overnight fast of at least 12 hours, the first venous blood sample was collected at 08:30 hour from the antecubital vein, while the second sample was collected two hours after $75 \mathrm{~g}$ oral glucose loading. The first blood sample was analyzed for baseline metabolic profiles, namely, fasting plasma glucose (FPG), total cholesterol, triglycerides (TG), high density lipoprotein-cholesterol (HDL-C), low density lipoprotein-cholesterol (LDL-C), and hormonal profile fasting insulin (FI). The second blood sample was analyzed for plasma glucose.

Insulin resistance was diagnosed using three indirect methods, namely, fasting insulin in excess of $25 \mu \mathrm{U} / \mathrm{ml}$, the fasting glucose to fasting insulin ratio lower than 4.5 [11], and the homeostasis model assessment-insulin resistance (HOMA-IR) calculated using Matthew's formula $\geq 2$ [12] [13].

The abnormal glucose metabolism was classified according to the World Health Organization (WHO) criteria [14] as follows: 1) Impaired fasting glucose (IFG) levels $\geq 110 \mathrm{mg} / \mathrm{dl}$ and $<126 \mathrm{mg} / \mathrm{dl}(6.1-6.9 \mathrm{mmol} / \mathrm{l}) ; 2)$ Impaired glucose tolerance (IGT) levels, that is, 2 -hr plasma glucose $\geq 140$ and $<200 \mathrm{mg} / \mathrm{dl}$ ( 7.8 $11.1 \mathrm{mmol} / \mathrm{l})$; and 3) Type 2 diabetes mellitus (T2DM), that is, FPG $\geq 126 \mathrm{mg} / \mathrm{dl}$ or 2-hr plasma glucose $\geq 200 \mathrm{mg} / \mathrm{dl}$.

Dyslipidemia was defined as total cholesterol $\geq 200 \mathrm{mg} / \mathrm{dl}$, triglycerides $\geq 150$ $\mathrm{mg} / \mathrm{dl}$, HDL-C $<50 \mathrm{mg} / \mathrm{dl}$, or LDL-C $\geq 160 \mathrm{mg} / \mathrm{dl}$, according to National Cholesterol Education Program Adult Treatment Panel (NCEP ATP) III, 2002 report [6] [15]. According to the definition recommended by the International Diabetes Federation (IDF), someone has metabolic syndrome if he/she has a raised blood pressure: a systolic blood pressure $\geq 130 \mathrm{mmHg}$ or a diastolic blood pressure $\geq$ $85 \mathrm{mmHg}$ or treatment of previously diagnosed hypertension [16].

Obesity was assessed by estimating body mass index (BMI, weight $/$ height $^{2}$, kilogram per $\mathrm{m}^{2}$ ). The BMI categories include underweight $<18.5$; normal weight 18.5 - 24.9; overweight 25 - 29.9; and obesity $\geq 30.0$ [14].

Plasma glucose levels were assayed using the standard glucose oxidase method, serum total cholesterol and triglycerides were assayed using the enzymatic method, and serum HDL-C levels were assayed using the precipitant method. All methods were adapted for use in the CHEMWELL autoanalyzer (Model 2902), AWARENESS TECHNOLOGY, Inc. Palm City, FL.USA. All kits for the assay were commercially available kits from RANDOX, USA. Methods for hormone analysis were described in a previous study [10]. 
Data analysis. Statistical analysis was performed using SPSS version 22 for Windows (SPSS Inc., Chicago, IL, USA). The normality of distribution of all continuous variables was checked using the Kolmogorov-Smirnov test. Data were presented in mean and standard deviation (SD), number (n) and percent (\%), or odds ratios (ORs) and 95\% confidence intervals (CI), as appropriate. Descriptive statistics were generated to enable comparisons between groups. Student $\mathrm{t}$-test was used to compare HOMA-IR categorical data. Multivariate logistic regression analysis was performed to identify the significant potential risk factors for IR. Hosmer and Lemeshow test was used to check for the goodness of fit of the model. A Statistical significance was considered to exist when P-value was $<0.05$.

\section{Ethical Consideration}

The Ethics and Research Committee of the UBTH approved the study proposal and issued the clearance certificate (ADM/E.22 A/VOL.VII/174). Fully informed consent was obtained from all women who participated in the study. They were assured of the confidentiality of information obtained, and that the results will be used for research only and not for any other purpose.

\section{Results}

A total of 116 Nigerian women with PCOS who formed the subject of this study were categorized according to BMI, with normal-weight 41 (35.3\%), overweight 54 (46.6), and obese 21 (18.1). Of these 116 women, 42 (36.2\%) were diagnosed with insulin resistance (HOMA-IR $\geq 2$ ), while $74(63.8 \%)$ were not insulin resistant. The 42 insulin resistant women were also categorized according to BMI and it revealed that 15 (35.7\%) women have normal-weight, 17 (40.5\%) women are overweight, and $10(23.8 \%)$ women are obese.

The age, anthropometric, blood pressure and biochemical characteristics of the 116 PCOS women of reproductive age are shown in Table 1 . The mean age was $32.05 \pm 5.36$ years, mean BMI was $26.77 \pm 3.85 \mathrm{~kg} / \mathrm{m}^{2}$, mean arterial blood pressure (MABP), $91.29 \pm 10.94 \mathrm{mmHg}$, mean fasting insulin, $10.69 \pm 11.26$ $\mu \mathrm{U} / \mathrm{ml}$, mean fasting plasma glucose to fasting insulin ratio was $18.34 \pm 25.03$ $\mathrm{mg} / \mu \mathrm{U}$, mean HOMA-IR was $2.21 \pm 2.46$ and mean TT was $1.34 \pm 1.45 \mathrm{ng} / \mathrm{ml}$. From the mean age and BMI, the 116 PCOS women are relatively young and overweight.

Table 2 compares the characteristics of the insulin resistant (HOMA-IR $\geq 2$ ) and the non-insulin resistant women in the groups against some independent parameters. The mean FPG showed a highly statistically significant increase $(\mathrm{P}=$ $0.001)$, and likewise the mean of 2 -hour post-OGTT glucose and FI $(\mathrm{P}<0.001)$ when compared to non-insulin resistant women, whereas the mean FPG:FI ratio showed a highly statistically significant decrease $(\mathrm{P}<0.001)$ compared to the non-insulin resistant group. Moreover, the mean levels of total cholesterol, triglycerides, LDL-C, TT, and LH:FSH ratio were elevated but not statistically sig- 
nificant when compared to the non-insulin resistant group. The mean HDL-C levels did not differ between each group. The greater abnormalities were found in glucose and lipid metabolism among women with IR than women without IR, which was expected.

Table 3 shows the prevalence of IR was $36.2 \%(n=42)$ among the 116 PCOS women. Abnormal carbohydrate metabolism also showed the following: two (1.7\%) women had IFG levels ( $\geq 110 \mathrm{mg} / \mathrm{dl})$, five (4.3\%) women had abnormal 2-hour glucose concentration $(\geq 140 \mathrm{mg} / \mathrm{dl})$ in the OGTT, three with IGT (2.6\%) and two with DM (1.7\%). Hyperinsulinemia was observed in nine (7.8\%) women.

The multivariate logistic regression analysis shown in Table 4 indicates that the independent risk factors for IR include, total cholesterol (OR, 1.07; 95\% CI, 1.04 - 1.10), triglycerides (OR, 1.08; 95\% CI, 1.04 - 1.13), LDL-C (OR, 1.08; 95\% CI, 1.04 - 1.12), FPG (OR, 1.11; 95\% CI, 1.06 - 1.16) and 2-hour OGTT (OR, 1.29; 95\% CI, 1.24 - 1.33). However, age, BMI, SBP, DBP were not significantly elevated and HDL-C was not significantly reduced. The multivariate logistic regression analysis in Table 5 predicts a statistically significant association of obesity with risk factors of IR and include age, SBP, DBP, total cholesterol, triglycerides, LDL-C, FPG, and 2-hour OGTT. There were a relative reduction of HDL-C levels in obese women (OR, 0.96; 95\% CI, 0.89 - 1.03) and overweight women (OR, 0.98; 95\% CI, 0.93 - 1.04) compared to normal-weight women, but this difference was not significant $(\mathrm{P}=0.240$ and $\mathrm{P}=0.506$ respectively). The Hosmer and Lemeshow test indicated that the model was fit $(\mathrm{P}=0.953)$.

Table 1. Characteristics of the 116 Nigerian women with polycystic ovary syndrome.

\begin{tabular}{cc}
\hline Characteristics & Mean \pm SD \\
\hline Age (years) & $32.05 \pm 5.36$ \\
Height $(\mathrm{m})$ & $1.62 \pm 0.06$ \\
Weight $(\mathrm{Kg})$ & $70.51 \pm 12.0$ \\
BMI $\left(\mathrm{Kg} / \mathrm{m}^{2}\right)$ & $26.77 \pm 3.85$ \\
SBP $(\mathrm{mmHg})$ & $119.22 \pm 13.52$ \\
DBP $(\mathrm{mmHg})$ & $77.33 \pm 11.06$ \\
MBP $(\mathrm{mmHg})$ & $116.73 \pm 14.18$ \\
MABP $(\mathrm{mmHg})$ & $91.29 \pm 10.94$ \\
FPG $(\mathrm{mg} / \mathrm{dl})$ & $81.54 \pm 14.82$ \\
2-hour post-OGTT glucose $(\mathrm{mg} / \mathrm{dl})$ & $97.35 \pm 26.81$ \\
FI $(\mu \mathrm{U} / \mathrm{ml})$ & $10.69 \pm 11.26$ \\
FPG:FI & $18.34 \pm 25.03$ \\
HOMA-IR & $2.21 \pm 2.46$ \\
Total Cholesterol (mg/dl) & $171.45 \pm 34.11$ \\
Triglycerides $(\mathrm{mg} / \mathrm{dl})$ & $80.55 \pm 29.67$ \\
HDL-C (mg/dl) & $48.99 \pm 15.72$ \\
LDL-C $(\mathrm{mg} / \mathrm{dl})$ & $106.36 \pm 33.04$ \\
\hline & \\
\hline &
\end{tabular}




\section{Continued}

$\begin{array}{cc}\text { TT (ng/ml) } & 1.34 \pm 1.45 \\ \text { LH:FSH } & 2.88 \pm 2.73 \\ \text { FSH (IU/L) } & 7.97 \pm 4.20 \\ \text { LH (IU/L) } & 19.10 \pm 16.11\end{array}$

Values are the mean \pm SD. Abbreviations: BMI, body mass index; DBP, diastolic blood pressure; FI, fasting insulin; FPG, fasting plasma glucose; FPG:FI, fasting plasma glucose to fasting insulin ratio; FSH, follicle stimulating hormone; HOMA-IR, homeostatic model assessment-insulin resistance; HDL-C, high density lipoprotein-cholesterol; $\mathrm{kg} / \mathrm{m}^{2}$, kilogram per meter squared; LDL-C, low density lipoprotein-cholesterol; LH, luteinizing hormone; LH:FSH, luteinizing hormone to follicle stimulating hormone ratio; MABP, mean arterial blood pressure; MBP, mean blood pressure; OGTT, oral glucose tolerance test; SBP, systolic blood pressure; TT, total testosterone.

Table 2. Comparison of the characteristics of 116 PCOS subjects with respect to their homeostasis model assessment of insulin resistance (HOMA-IR) using t-test.

\begin{tabular}{|c|c|c|c|}
\hline Risk factors & $\begin{array}{l}\text { Non-insulin resistant } \\
(\text { HOMA-IR }<2), \mathrm{n}=74\end{array}$ & $\begin{array}{c}\text { Insulin-resistant } \\
(\text { HOMA-IR } \geq 2), \mathrm{n}=42\end{array}$ & P-value \\
\hline Age (years) & $32.15 \pm 5.23$ & $31.88 \pm 5.65$ & 0.797 \\
\hline Height (m) & $1.62 \pm 0.06$ & $1.63 \pm 0.07$ & 0.188 \\
\hline Weight (Kg) & $69.34 \pm 10.96$ & $72.58 \pm 13.54$ & 0.163 \\
\hline $\mathrm{BMI}\left(\mathrm{kg} / \mathrm{m}^{2}\right)$ & $26.54 \pm 3.77$ & $27.16 \pm 4.01$ & 0.407 \\
\hline $\mathrm{SBP}(\mathrm{mmHg})$ & $118.11 \pm 14.68$ & $121.19 \pm 11.09$ & 0.240 \\
\hline $\mathrm{DBP}(\mathrm{mmHg})$ & $76.62 \pm 11.26$ & $78.57 \pm 10.72$ & 0.364 \\
\hline MBP (mmHg) & $115.45 \pm 14.56$ & $118.97 \pm 13.36$ & 0.201 \\
\hline MABP (mmHg) & $90.45 \pm 11.47$ & $92.78 \pm 9.90$ & 0.273 \\
\hline FPG (mg/dl) & $78.22 \pm 10.85$ & $87.4 \pm 18.75$ & $0.001^{* *}$ \\
\hline $\begin{array}{l}\text { 2-hour post-OGTT } \\
\text { glucose }(\mathrm{mg} / \mathrm{dl})\end{array}$ & $87.77 \pm 12.13$ & $114.24 \pm 36.00$ & $<0.001^{* *}$ \\
\hline $\mathrm{FI}(\mu \mathrm{U} / \mathrm{ml})$ & $5.04 \pm 2.58$ & $20.65 \pm 13.59$ & $<0.001^{* *}$ \\
\hline FPG:FI (mg/ $\mu \mathrm{U})$ & $25.42 \pm 28.86$ & $5.87 \pm 4.87$ & $<0.001^{* *}$ \\
\hline $\begin{array}{l}\text { Total cholesterol } \\
\qquad(\mathrm{mg} / \mathrm{dl})\end{array}$ & $167.07 \pm 28.41$ & $179.17 \pm 41.60$ & 0.066 \\
\hline Triglycerides (mg/dl) & $77.59 \pm 26.81$ & $85.76 \pm 33.85$ & 0.155 \\
\hline $\mathrm{HDL}-\mathrm{C}(\mathrm{mg} / \mathrm{dl})$ & $48.14 \pm 12.62$ & $50.5 \pm 20.14$ & 0.438 \\
\hline LDL-C (mg/dl) & $103.45 \pm 28.00$ & $111.5 \pm 40.30$ & 0.208 \\
\hline $\mathrm{TT}(\mathrm{ng} / \mathrm{ml})$ & $1.28 \pm 1.09$ & $1.45 \pm 1.94$ & 0.528 \\
\hline LH:FSH & $2.75 \pm 2.49$ & $3.11 \pm 3.13$ & 0.498 \\
\hline FSH (IU/l) & $7.88 \pm 4.14$ & $8.12 \pm 4.36$ & 0.774 \\
\hline LH (IU/l) & $17.68 \pm 13.57$ & $21.59 \pm 19.75$ & 0.211 \\
\hline
\end{tabular}

${ }^{*} \mathrm{P}<0.01$ was highly significant; ${ }^{*} \mathrm{P}<0.05$ was significant; Values are the mean $\pm \mathrm{SD}$. Abbreviations: $\mathrm{BMI}$, body mass index; DBP, diastolic blood pressure; FI, fasting insulin; FPG, fasting plasma glucose; FPG:FI, fasting plasma glucose to fasting insulin ratio; FSH, follicle stimulating hormone; HOMA-IR, homeostatic model assessment-insulin resistance; HDL-C, high density lipoprotein-cholesterol; LDL-C, low density lipoprotein-cholesterol; $\mathrm{LH}$, luteinizing hormone; LH:FSH, luteinizing hormone to follicle stimulating hormone ratio; $\mathrm{MABP}$, mean arterial blood pressure; $\mathrm{MBP}$, mean blood pressure; OGTT, oral glucose tolerance test; SBP, systolic blood pressure; TT, total testosterone. 
Table 3. Prevalence of insulin resistance and abnormal carbohydrate metabolism in 116 Nigerian women with polycystic ovary syndrome.

\begin{tabular}{ccc}
\hline Characteristics & $\mathrm{N}$ & $\%(95 \% \mathrm{CI})$ \\
\hline Insulin resistance (HOMA-IR $\geq 2)$ & 42 & $36.2(26.6-46.2)$ \\
Impaired fasting glucose & 2 & $1.7(0.97-2.03)$ \\
Impaired glucose tolerance & 3 & $2.6(1.97-3.03)$ \\
$\mathrm{DM}$ & 2 & $1.7(0.97-2.03)$ \\
Hyperinsulinemia $(\mathrm{FI} \geq 25 \mu \mathrm{U} / \mathrm{ml})$ & 9 & $7.8(6.82-8.78)$ \\
\hline
\end{tabular}

Abbreviations: 95\% CI, 95 percent confidence intervals; DM, diabetes mellitus; FI, fasting insulin; HOMA-IR, homeostatic model assessment-insulin resistance.

Table 4. Multivariate logistic regression analysis for risk factors of IR in women with PCOS.

\begin{tabular}{|c|c|c|c|}
\hline Variables & HOMA-IR & OR $(95 \% \mathrm{CI})$ & $\mathrm{P}$-value \\
\hline \multirow[t]{2}{*}{ Age (years) } & $\geq 2(\mathrm{n}=42)$ & $0.99(0.92-1.06)$ & 0.683 \\
\hline & $<2(\mathrm{n}=74)$ & 1 & \\
\hline \multirow[t]{2}{*}{ Height (m) } & $\geq 2(\mathrm{n}=42)$ & $1.01(0.98-1.04)$ & 0.545 \\
\hline & $<2(\mathrm{n}=74)$ & 1 & \\
\hline \multirow[t]{2}{*}{ Weight (kg) } & $\geq 2(\mathrm{n}=42)$ & $1.09(0.98-1.21)$ & $<0.001$ \\
\hline & $<2(\mathrm{n}=74)$ & 1 & \\
\hline \multirow{2}{*}{ BMI $\left(\mathrm{kg} / \mathrm{m}^{2}\right)$} & $\geq 2(\mathrm{n}=42)$ & $1.04(0.88-1.24)$ & \\
\hline & $<2(\mathrm{n}=74)$ & 1 & $<0.001$ \\
\hline \multirow{2}{*}{$\mathrm{SBP}(\mathrm{mmHg})$} & $\geq 2(\mathrm{n}=42)$ & $1.023(0.99-1.06)$ & 0195 \\
\hline & $<2(\mathrm{n}=74)$ & 1 & 0.195 \\
\hline \multirow{2}{*}{ DBP (mmHg) } & $\geq 2(\mathrm{n}=42)$ & $1.019(0.98-1.06)$ & 0383 \\
\hline & $<2(\mathrm{n}=74)$ & 1 & 0.505 \\
\hline \multirow{2}{*}{ MBP (mmHg) } & $\geq 2(\mathrm{n}=42)$ & $0.96(0.91-1.02)$ & 0175 \\
\hline & $<2(\mathrm{n}=74)$ & 1 & 0.175 \\
\hline \multirow{2}{*}{ MABP (mmHg) } & $\geq 2(\mathrm{n}=42)$ & $0.99(0.89-1.10)$ & 0839 \\
\hline & $<2(\mathrm{n}=74)$ & 1 & 0.039 \\
\hline \multirow{2}{*}{$\begin{array}{l}\text { Total cholesterol } \\
\qquad(\mathrm{mg} / \mathrm{dl})\end{array}$} & $\geq 2(\mathrm{n}=42)$ & $1.07(1.04-1.10)$ & $<0.001$ \\
\hline & $<2(\mathrm{n}=74)$ & 1 & $<0.001$ \\
\hline \multirow{2}{*}{ Triglycerides (mg/dl) } & $\geq 2(\mathrm{n}=42)$ & $1.08(1.04-1.13)$ & $<0001$ \\
\hline & $<2(\mathrm{n}=74)$ & 1 & $<0.001$ \\
\hline \multirow{2}{*}{ HDL-C (mg/dl) } & $\geq 2(\mathrm{n}=42)$ & $1.05(1.00-1.11)$ & 0.070 \\
\hline & $<2(\mathrm{n}=74)$ & 1 & 0.070 \\
\hline \multirow{2}{*}{ LDL-C (mg/dl) } & $\geq 2(\mathrm{n}=42)$ & $1.08(1.04-1.12)$ & $<0.001$ \\
\hline & $<2(\mathrm{n}=74)$ & 1 & $<0.001$ \\
\hline \multirow{2}{*}{ FPG (mg/dl) } & $\geq 2(\mathrm{n}=42)$ & $1.11(1.06-1.16)$ & $<0.001$ \\
\hline & $<2(\mathrm{n}=74)$ & 1 & $<0.001$ \\
\hline \multirow{2}{*}{$\begin{array}{l}\text { 2-hour post-OGTT } \\
\qquad(\mathrm{mg} / \mathrm{dl})\end{array}$} & $\geq 2(\mathrm{n}=42)$ & $1.29(1.24-1.33)$ & $<\cap 001$ \\
\hline & $<2(\mathrm{n}=74)$ & 1 & $<0.001$ \\
\hline \multirow{2}{*}{ HOMA-IR } & $\geq 2(\mathrm{n}=42)$ & $28.33(19.34-41.48)$ & $<0001$ \\
\hline & $<2(\mathrm{n}=74)$ & 1 & $<0.001$ \\
\hline
\end{tabular}

OR, odds ratio; 95\% CI, 95 percent confidence intervals. $\mathrm{P}<0.05$ was significant. Abbreviations: BMI, body mass index; DBP, diastolic blood pressure; FPG, fasting plasma glucose; HOMA-IR, homeostatic model assessment-insulin resistance; HDL-C, high density lipoprotein-cholesterol; $\mathrm{kg} / \mathrm{m}^{2}$, kilogram per meter squared; LDL-C, low density lipoprotein-cholesterol; MABP, mean arterial blood pressure; MBP, mean blood pressure; OGTT, oral glucose tolerance test; SBP, systolic blood pressure. 
Table 5. Multivariate logistic regression analysis of PCOS women with IR according to the BMI categories.

\begin{tabular}{|c|c|c|c|}
\hline Variables & BMI Categories & OR $(95 \% \mathrm{CI})$ & $\mathrm{P}$-value \\
\hline Age (years) & $\begin{array}{c}\text { Obese } \\
\text { Overweight } \\
\text { Normal-weight }\end{array}$ & $\begin{array}{c}1.21(1.10-1.32) \\
1.13(1.05-1.21) \\
1\end{array}$ & $\begin{array}{c}<0.001 \\
0.001\end{array}$ \\
\hline Height (m) & $\begin{array}{c}\text { Obese } \\
\text { Overweight } \\
\text { Normal-weight }\end{array}$ & $\begin{array}{c}1.01(0.97-1.05) \\
1.01(0.97-1.04) \\
1\end{array}$ & $\begin{array}{l}0.604 \\
0.722\end{array}$ \\
\hline Weight (kg) & $\begin{array}{c}\text { Obese } \\
\text { Overweight } \\
\text { Normal-weight }\end{array}$ & $\begin{array}{c}1.54(1.35-1.75) \\
1.18(1.05-1.33) \\
1\end{array}$ & $\begin{array}{c}<0.001 \\
0.008\end{array}$ \\
\hline BMI $\left(\mathrm{kg} / \mathrm{m}^{2}\right)$ & $\begin{array}{c}\text { Obese } \\
\text { Overweight } \\
\text { Normal-weight }\end{array}$ & $\begin{array}{c}1.39(1.14-1.71) \\
1.18(0.97-1.43) \\
1\end{array}$ & $\begin{array}{l}0.001 \\
0.097\end{array}$ \\
\hline SBP (mmHg) & $\begin{array}{c}\text { Obese } \\
\text { Overweight } \\
\text { Normal-weight }\end{array}$ & $\begin{array}{c}1.12(1.07-1.17) \\
1.08(1.04-1.12) \\
1\end{array}$ & $\begin{array}{l}<0.001 \\
<0.001\end{array}$ \\
\hline $\mathrm{DBP}(\mathrm{mmHg})$ & $\begin{array}{c}\text { Obese } \\
\text { Overweight } \\
\text { Normal-weight }\end{array}$ & $\begin{array}{c}1.17(1.10-1.24) \\
1.09(1.04-1.14) \\
1\end{array}$ & $\begin{array}{l}<0.001 \\
<0.001\end{array}$ \\
\hline MBP (mmHg) & $\begin{array}{c}\text { Obese } \\
\text { Overweight } \\
\text { Normal-weight }\end{array}$ & $\begin{array}{c}1.05(0.97-1.13) \\
1.00(0.93-1.07) \\
1\end{array}$ & $\begin{array}{l}0.266 \\
0.987\end{array}$ \\
\hline MABP (mmHg) & $\begin{array}{c}\text { Obese } \\
\text { Overweight } \\
\text { Normal-weight }\end{array}$ & $\begin{array}{c}1.11(0.98-1.25) \\
1.04(0.95-1.14) \\
1\end{array}$ & $\begin{array}{l}0.098 \\
0.377\end{array}$ \\
\hline $\begin{array}{l}\text { Total cholesterol } \\
\qquad(\mathrm{mg} / \mathrm{dl})\end{array}$ & $\begin{array}{c}\text { Obese } \\
\text { Overweight } \\
\text { Normal-weight }\end{array}$ & $\begin{array}{c}1.08(1.03-1.12) \\
1.07(1.04-1.11) \\
1\end{array}$ & $\begin{array}{l}<0.001 \\
<0.001\end{array}$ \\
\hline Triglycerides (mg/dl) & $\begin{array}{c}\text { Obese } \\
\text { Overweight } \\
\text { Normal-weight }\end{array}$ & $\begin{array}{c}1.34(1.27-1.42) \\
1.05(1.00-1.10) \\
1\end{array}$ & $\begin{array}{c}<0.001 \\
0.054\end{array}$ \\
\hline HDL-C (mg/dl) & $\begin{array}{c}\text { Obese } \\
\text { Overweight } \\
\text { Normal-weight }\end{array}$ & $\begin{array}{c}0.96(0.89-1.03) \\
0.98(0.93-1.04) \\
1\end{array}$ & $\begin{array}{l}0.240 \\
0.506\end{array}$ \\
\hline LDL-C (mg/dl) & $\begin{array}{c}\text { Obese } \\
\text { Overweight } \\
\text { Normal-weight }\end{array}$ & $\begin{array}{c}1.10(1.04-1.15) \\
1.12(1.08-1.17) \\
1\end{array}$ & $\begin{array}{c}0.001 \\
<0.001\end{array}$ \\
\hline $\mathrm{TT}(\mathrm{ng} / \mathrm{ml})$ & $\begin{array}{c}\text { Obese } \\
\text { Overweight } \\
\text { Normal-weight }\end{array}$ & $\begin{array}{c}1.01 \quad(0.22-4.47) \\
1.14(0.34-3.80) \\
1\end{array}$ & $\begin{array}{l}1.000 \\
0.827\end{array}$ \\
\hline LH (IU/l) & $\begin{array}{c}\text { Obese } \\
\text { Overweight } \\
\text { Normal-weight }\end{array}$ & $\begin{array}{c}0.63(0.47-0.84) \\
0.98(0.74-1.30) \\
1\end{array}$ & $\begin{array}{l}0.001 \\
0.885\end{array}$ \\
\hline LH:FSH & $\begin{array}{c}\text { Obese } \\
\text { Overweight } \\
\text { Normal-weight }\end{array}$ & $\begin{array}{c}0.79(0.29-2.10) \\
1.10(0.40-3.05) \\
1\end{array}$ & $\begin{array}{l}0.631 \\
0.857\end{array}$ \\
\hline FPG (mg/dl) & $\begin{array}{c}\text { Obese } \\
\text { Overweight } \\
\text { Normal-weight }\end{array}$ & $\begin{array}{c}1.15(1.08-1.21) \\
1.05(1.00-1.10) \\
1\end{array}$ & $\begin{array}{c}<0.001 \\
0.055\end{array}$ \\
\hline
\end{tabular}


Continued

\begin{tabular}{cccc}
\hline \multirow{2}{*}{$\begin{array}{c}\text { 2-hour post-OGTT } \\
(\mathrm{mg} / \mathrm{dl})\end{array}$} & Obese & $1.22(1.16-1.29)$ & $<0.001$ \\
& Overweight & $1.06(1.02-1.11)$ & 0.006 \\
& Normal-weight & 1 & \\
\multirow{2}{*}{ HOMA-IR } & Obese & $2.41(1.42-4.09)$ & 0.001 \\
& Overweight & $1.29(0.86-1.94)$ & 0.210 \\
& Normal-weight & 1 & \\
\hline
\end{tabular}

OR, odds ratio; 95\% CI, 95 percent confidence intervals. P < 0.05 was significant. Abbreviations: BMI, body mass index; DBP, diastolic blood pressure; FPG, fasting plasma glucose; HOMA-IR, homeostatic model assessment-insulin resistance; HDL-C, high density lipoprotein-cholesterol; $\mathrm{kg} / \mathrm{m}^{2}$, kilogram per meter squared; LDL-C, low density lipoprotein-cholesterol; LH, luteinizing hormone; LH:FSH, luteinizing hormone to follicle stimulating hormone ratio; $\mathrm{MABP}$, mean arterial blood pressure; MBP, mean blood pressure; OGTT, oral glucose tolerance test; $\mathrm{SBP}$, systolic blood pressure; TT, total testosterone.

\section{Discussion}

This study was undertaken to establish the prevalence of insulin resistance among infertile women with PCOS in our region and to determine the risk factors associated with IR in PCOS women. This is the first study on the prevalence of insulin resistance in Nigerian women with PCOS to the best of our knowledge. The etiology of IR in women with PCOS, although intensively studied, is not entirely clear. The mechanisms involve a unique disorder of insulin action secondary to decreased insulin receptor signaling, likely caused by serine hyperphosphorylation of the receptor and of the insulin receptor substrate 1 [3]. The various techniques used for the diagnosis of IR include FPG, OGTT, glucose/insulin ratio, HOMA-IR, quantitative insulin sensitivity check index (QUICKI), and the gold standard which is the hyperinsulinemic-euglycemic clamp [3] [17] [18]. However, Wanderley et al. (2018) [9], analyzed the frequency of IR in Portuguese PCOS women using four of the indirect methods and reported no statistical difference among the four methods with regards to the frequency of IR. In this study, IR was determined using the HOMA-IR technique and the prevalence rate obtained among infertile Nigerian PCOS women was $36.2 \%$. Several studies have reported higher prevalence rates of IR ranging from $44 \%$ to $70 \%$ among PCOS women in the US, $60.2 \%$ in Mexican women and $50.04 \%$ in Portuguese women [3] [8] [9] when compared to this study. However, lower prevalence rates were reported in Iranian (24.3\%) and Thai (20.0\%) women [5] [6] than observed in this study. The great variability observed in the prevalence rates might be attributable to the diagnostic criteria used in PCOS, different PCOS phenotypes, ethnic differences in insulin action, environmental factors such as diet, or the method used in identifying IR [3]. Some of these factors might have contributed significantly to the prevalence of IR in our region.

The prevalence of IGT and DM found in this study was low in contrast to that reported among US women with PCOS which ranged from $23 \%$ and $35 \%$ for IGT and between $4 \%$ and $10 \%$ for DM [19] [20] [21]. The prevalence rates reported in Thai (13.6\%) [6], Australia (15.6\%) [22], the Mediterranean region (15.7\%) [23], Hispanics (22.1\%) [8] and China (20.5\%) [24] were still higher than the findings in this study. These different findings are suggestive of the ef- 
fects of environmental factors and ethnicity, as also observed in the prevalence rates reported among Korean PCOS women [25].

Findings from PCOS women in this study demonstrated the presence of IR across the BMI categories, with the average frequency of $35.7 \%$ found in normal-weight, $40.5 \%$ found in overweight and $23.8 \%$ found in obese women. However, this was not consistent with the report of Wanderley et al., 2018 [9] which showed the average frequency of IR as $20.31 \%$ in normal-weight, $50 \%$ in overweight and $76.59 \%$ in obese women. Other studies have observed that in the presence of obesity, IR frequency and magnitude increases [1] [4] [26]. In the present study, the findings showed that women are overweight, and the overweight category had the highest IR frequency. Moreover, there was a significant association of IR with obesity and dyslipidemia which is consistent with other reports [7] [9], and the association of IR with total cholesterol, triglycerides, and LDL-C reflected the effect of dyslipidemia for PCOS women, which might be suggestive of cardiovascular risk. Furthermore, the levels of FPG and 2-hour OGTT glucose are also adequate risk factors for IR in PCOS women in our region, especially in the presence of obesity. Some studies [27] suggested that IR could contribute to dyslipidemia frequently observed in PCOS women through various mechanisms, thus corroborating the findings in this study.

Some limitations in this study are worth mentioning and include the method used in the diagnosis of IR. Whereas the gold standard is the hyperinsulinemic-euglycemic clamp, it is quite elaborate in clinical practice. Nevertheless, the HOMA-IR which is an indirect method used in this study has been reported to have a good correlation with clamp technique [28]. Results obtained in this study included only PCOS women with infertility, therefore the outcomes are not generalizable to PCOS women without infertility. Additionally, this was not a population-based sample, as such further research is needed in this regard. However, findings in this study cannot be ignored as it has shown the prevalence of IR and its significant association with obesity and dyslipidemia in this region. A further study to compare IR among age-matched and BMI-matched non-PCOS women and those with PCOS would be necessary.

\section{Conclusion}

In conclusion, the findings obtained in the present study indicate that women with PCOS and IR in our population are associated with risk factors like obesity and dyslipidemia as observed in other regions, and are equally prone to cardiovascular risks. However, the prevalence of IGT was found to be low compared to other countries. Therefore, early detection, treatment, and appropriate lifestyle modifications could improve fertility, quality of life, and reduce the risk of PCOS long term complications.

\section{Acknowledgements}

The authors acknowledge Isaac Adebanjo, the Statistician for his diligence. 


\section{Conflicts of Interest}

The authors declare no conflicts of interest regarding the publication of this paper.

\section{References}

[1] Baldani, D.P., Skrgatic, L. and Ougouag, R. (2015) Polycystic Ovary Syndrome: Important Underrecognised Cardiometabolic Risk Factor in Reproductive-Age Women. International Journal of Endocrinology, 2015, Article ID: 786362. https://doi.org/10.1155/2015/786362

[2] Wild, R.A. (2002) Long-Term Health Consequences of PCOS. Human Reproduction Update, 8, 231-241. https://doi.org/10.1093/humupd/8.3.231

[3] Diamanti-Kandarakis, E. and Dunaif, A. (2012) Insulin Resistance and the Polycystic Ovary Syndrome Revisited: An Update on Mechanisms and Implications. Endocrinology Reviews, 33, 981-1030. https://doi.org/10.1210/er.2011-1034

[4] Laganá, A.S., Rossetti, P., Buscema, M., Vignera, S.L., Condorelli, R.A., Gullo, G., Granese, R. and Triolo, O. (2016) Metabolism and Ovarian Function in PCOS Women: A Therapeutic Approach with Inositols. International Journal of Endocrinology, 2016, Article ID: 6306410. https://doi.org/10.1155/2016/6306410

[5] Mehrabian, F., Khani, B., Kelishadi, R. and Kermani, N. (2011) The Prevalence of Metabolic Syndrome and Insulin Resistance According to the Phenotypic Subgroups of Polycystic Ovary Syndrome in a Representative Sample of Iranian Females. Journal of Research in Medical Science, 16, 763-769.

[6] Wongwananuruk, T., Rattanachaiyanont, M., Indhavivadhana, S., Leerasiri, P., Techatraisak, K., Tanmahasamut, P., Angsuwathana, S. and Dangrat, C. (2012) Prevalence and Clinical Predictors of Insulin Resistance in Reproductive-Aged Thai Women with Polycystic Ovary Syndrome. International Journal of Endocrinology, 2012, Article ID: 529184. https://doi.org/10.1155/2012/529184

[7] Le, M.T., Nguyen, V.Q.H., Truong, Q.V., Le, D.D., Le, V.N.S. and Cao, N.T. (2018) Metabolic Syndrome and Insulin Resistance Syndrome among Infertile Women with Polycystic Ovary Syndrome: A Cross-Sectional Study from Central Vietnam. Endocrinology and Metabolism, 33, 447-458. https://doi.org/10.3803/EnM.2018.33.4.447

[8] Reyes-Munoz, E., Ortega-Gonzalez, C., Martinez-Cruz, N., Arce-Sanchez, L., Estrada-Gutierrez, G., Moran, C., Sanchez-Serrano, A.P., Higareda-Sanchez, R. and Jara-Diaz, F. (2016) Association of Obesity and Overweight with the Prevalence of Insulin Resistance, Pre-Diabetes and Clinical-Biochemical Characteristics among Infertile Mexican Women with Polycystic Ovary Syndrome: A Cross-Sectional Study. BMJ Open, 6, e012107. https://doi.org/10.1136/bmjopen-2016-012107

[9] Wanderley, M., Pereira, L.C.R., Santos, C.B., Santos da Cunha, V. and Neves, M.V.J. (2018) Association between Insulin Resistance and Cardiovascular Risk Factors in Polycystic Ovary Syndrome Patients. Revista Brasileira de Ginecologia e Obstetrícia, 40, 188-195. https://doi.org/10.1055/s-0038-1642634

[10] Akpata, C.B., Uadia, P.O. and Okonofua, F.E. (2018) Prevalence of Polycystic Ovary Syndrome in Nigerian Women with Infertility: A Prospective Study of the Three Assessment Criteria. Open Journal of Obstetrics and Gynecology, 8, 1109-1120. https://doi.org/10.4236/ojog.2018.812112

[11] Lin, T.C., Yen, J.M., Gong, K.B., Kuo, T.C., Ku, D.C., Liang, S.F. and Wu, M.J. (2006) Abnormal Glucose Tolerance and Insulin Resistance in Polycystic Ovary 
Syndrome amongst the Taiwanese Population-Not Correlated with Insulin Receptor Substrate-1 Gly972Arg/Ala513Pro Polymorphism. BMC Medical Genetics, 7, 36-38. https://doi.org/10.1186/1471-2350-7-36

[12] Matthews, D.R., Hosker, J.P., Rudenski, A.S., Naylor, B.A., Treacher, D.F. and Turner, R.C. (1985) Homeostasis Model Assessment: Insulin Resistance and $\beta$-Cell Function from Fasting Plasma Glucose and Insulin Concentrations in Man. Diabetologia, 28, 412-419. https://doi.org/10.1007/BF00280883

[13] Jensterle, M., Weber, M., Pfeifer, M., Prezelj, J., Pfutzner, A. and Janez, A. (2008) Assessment of Insulin Resistance in Young Women with Polycystic Ovary Syndrome. International Journal of Gynecology and Obstetrics, 102, 137-140. https://doi.org/10.1016/j.ijgo.2008.03.017

[14] World Health Organization (2006) BMI Classification. Global Database on Body Mass Index.

[15] (2002) Third Report of the National Cholesterol Education Program (NCEP) Expert Panel on Detection, Evaluation, and Treatment of High Blood Cholesterol in Adults (Adult Treatment Panel III) Final Report. Circulation, 106, 3143-3421. https://doi.org/10.1161/circ.106.25.3143

[16] Ford, E.S. (2005) Prevalence of the Metabolic Syndrome Defined by the International Diabetes Federation among Adults in the U.S. Diabetes Care, 28, 2745-2749. https://doi.org/10.2337/diacare.28.11.2745

[17] Carmina, E. and Lobo, R.A. (2004) Use of Fasting Blood to Assess the Prevalence of Insulin Resistance in Women with Polycystic Ovary Syndrome. Fertility and Sterility, 82, 661-665. https://doi.org/10.1016/j.fertnstert.2004.01.041

[18] Mather, K. (2009) Surrogate Measures of Insulin Resistance: of Rats, Mice, and Men. American Journal of Physiology — Endocrinology Metabolism, 296, E398-E399. https://doi.org/10.1152/ajpendo.90889.2008

[19] Legro, R.S., Kunselman, A.R., Dodson, W.C. and Dunaif, A. (1999) Prevalence and Predictors of Risk for Type 2 Diabetes Mellitus and Impaired Glucose Tolerance in Polycystic Ovary Syndrome: A Prospective, Controlled Study in 254 Affected Women. Journal of Clinical Endocrinology and Metabolism, 84, 165-169.

[20] Ehrmann, D.A., Barnes, R.B., Rosenfield, R.L., Cavaghan, M.K. and Imperial, J. (1999) Prevalence of Impaired Glucose Tolerance and Diabetes in Women with Polycystic Ovary Syndrome. Diabetes Care, 22, 141-146. https://doi.org/10.2337/diacare.22.1.141

[21] Ehrmann, D.A., Kasza, K., Azziz, R., Legro, R.S. and Ghazzi, M.N. (2005) Effects of Race and Family History of Type 2 Diabetes on the Metabolic Status of Women with Polycystic Ovary Syndrome. Journal of Clinical Endocrinology and Metabolism, 90, 66-71. https://doi.org/10.1210/jc.2004-0229

[22] Dabadghao, P., Roberts, B.J., Wang, J., Davies, M.J. and Norman, R.J. (2007) Glucose Tolerance Abnormalities in Australian Women with Polycystic Ovary Syndrome. Medical Journal of Australia, 187, 328-331.

[23] Gambineri, A., Pelusi, C., Manicardi, E., Vicennati, V., Cacciari, M., Morselli-Labate, A.M., Pagotto, U. and Pasquali, R. (2004) Glucose Intolerance in a Large Cohort of Mediterranean Women with Polycystic Ovary Syndrome: Phenotype and Associated Factors. Diabetes, 53, 2353-2358. https://doi.org/10.2337/diabetes.53.9.2353

[24] Chen, X., Yang, D., Li, L., Feng, S. and Wang, L. (2006) Abnormal Glucose Tolerance in Chinese Women with Polycystic Ovary Syndrome. Human Reproduction, 21, 2027-2032. https://doi.org/10.1093/humrep/del142 
[25] Lee, H., Oh, J.Y., Sung, Y.A. and Chung, H. (2009) The Prevalence and Risk Factors for Glucose Intolerance in Young Korean Women with Polycystic Ovary Syndrome. Endocrine, 36, 326-332. https://doi.org/10.1007/s12020-009-9226-7

[26] Moran, C., Arriaga, M., Rodriguez, G. and Moran, S. (2012) Obesity Differentially Affects Phenotypes of Polycystic Ovary Syndrome. International Journal of Endocrinology, 2012, Article ID: 317241. https://doi.org/10.1155/2012/317241

[27] Wild, R.A., Rizzo, M., Clifton, S. and Carmina, E. (2011) Lipid Levels in Polycystic Ovary Syndrome: Systematic Review and Meta-Analysis. Fertility and Sterility, 95, 1073-1079.e11. https://doi.org/10.1016/j.fertnstert.2010.12.027

[28] Otten, J., Ahren, B. and Olsson, T. (2014) Surrogate Measures of Insulin Sensitivity vs the Hyperinsulinaemic-Euglycaemic Clamp: A Meta-Analysis. Diabetologia, 57, 1781-1788. https://doi.org/10.1007/s00125-014-3285-x 